\title{
Ischemic postconditioning attenuates the inflammatory response in ischemia/reperfusion myocardium by upregulating miR-499 and inhibiting TLR2 activation
}

\author{
XIN-YUE ZHANG ${ }^{1}$, ZHENG HUANG $^{1}$, QING-JIE LI $^{2}$, GUO-QIANG ZHONG ${ }^{1,3,4}$, \\ JIAN-JUN MENG ${ }^{5}$, DONG-XIAO WANG ${ }^{1}$ and RONG-HUI TU ${ }^{3,4,6}$ \\ ${ }^{1}$ Department of Cardiology, First Affiliated Hospital, Guangxi Medical University; \\ ${ }^{2}$ Department of Cardiology, Second Affiliated Hospital, Guangxi Medical University; \\ ${ }^{3}$ Guangxi Key Laboratory of Precision Medicine in Cardio-Cerebrovascular Diseases Control and Prevention; \\ ${ }^{4}$ Guangxi Clinical Research Center for Cardio-Cerebrovascular Diseases, First Affiliated Hospital, \\ Guangxi Medical University; Departments of ${ }^{5}$ Geriatric Health Care Center and ${ }^{6}$ Geriatric Cardiology, \\ First Affiliated Hospital, Guangxi Medical University, Nanning, Guangxi 530021, P.R. China
}

Received September 9, 2019; Accepted March 25, 2020

DOI: $10.3892 / \mathrm{mmr} .2020 .11104$

\begin{abstract}
Toll-like receptor 2 (TLR2)-mediated myocardial inflammation serves an important role in promoting myocardial ischemic/reperfusion (I/R) injury. Previous studies have shown that miR-499 is critical for cardioprotection after ischemic postconditioning (IPostC). Therefore, the present study evaluated the protective effect of IPostC on the myocardium by inhibiting TLR2, and also assessed the involvement of microRNA (miR)-499. Rat hearts were subjected to $30 \mathrm{~min}$ of ischemia and $2 \mathrm{~h}$ of reperfusion. The IPostC was 3 cycles of $30 \mathrm{sec}$ of reperfusion and $30 \mathrm{sec}$ of re-occlusion prior to reperfusion. In total, 90 rats were randomly divided into six groups ( $\mathrm{n}=15$ per group): Sham; I/R; IPostC; miR-499 negative control adeno-associated virus (AAV) vectors + IPostC; miR-499 inhibitor AAV vectors + IPostC; and miR-499 mimic AAV vectors + IPostC. It was identified that IPostC significantly decreased the I/R-induced cardiomyocyte apoptotic index $(29.4 \pm 2.03 \%$ in IPostC vs. $42.64 \pm 2.27 \%$ in I/R; $\mathrm{P}<0.05)$ and myocardial infarct size $(48.53 \pm 2.49 \%$ in IPostC vs. $66.52 \pm 3.1 \%$ in $\mathrm{I} / \mathrm{R} ; \mathrm{P}<0.05)$. Moreover, these beneficial effects were accompanied by increased miR-499 expression levels (as demonstrated by reverse transcription-quantitative PCR) in the myocardial tissue and decreased TLR2, protein kinase $\mathrm{C}$ (PKC), interleukin (IL)-1 $\beta$ and IL-6 expression levels (as demonstrated by western blotting and ELISA)
\end{abstract}

Correspondence to: Dr Rong-Hui Tu, Guangxi Key Laboratory of Precision Medicine in Cardio-Cerebrovascular Diseases Control and Prevention, 22 Shuangyong Road, Nanning, Guangxi 530021, P.R. China

E-mail: lily072trh@sina.com

Key words: microRNA-499, ischemic postconditioning, toll-like receptor 2, protein kinase $\mathrm{C}$, inflammation in the myocardium and serum. The results indicated that IPostC + miR-499 mimics significantly inhibited inflammation and the PKC signaling pathway and enhanced the anti-inflammatory and anti-apoptotic effects of IPostC. However, IPostC + miR-499 inhibitors had the opposite effect. Therefore, it was speculated that IPostC may have a miR-499-dependent cardioprotective effect. The present results suggested that miR-499 may be involved in IPostC-mediated ischemic cardioprotection, which may occur via local and systemic TLR2 inhibition, subsequent inhibition of the PKC signaling pathway and a decrease in inflammatory cytokine release, including IL-1 $\beta$ and IL-6. Moreover, these effects will ultimately lead to a decrease in the myocardial apoptotic index and myocardial infarct size via the induction of the anti-apoptotic protein Bcl-2, and inhibition of the pro-apoptotic protein Bax in myocardium.

\section{Introduction}

Reperfusion is the most effective treatment for decreasing myocardial infarct size and myocardial apoptosis following ischemic injury (1). However, acting as a double-edged sword, reperfusion may initiate additional damage, such as reperfusion injury, which may counter the benefits of reperfusion and cause fatal damage in patients (2). Previous studies have shown that Toll-like receptor 2 (TLR2) triggers an inflammatory cascade and serves an important role in ischemic/reperfusion (I/R) injury (3-5). As part of the innate immune defense, TLR2 is able to recognize danger signals and can regulate endogenous or exogenous inflammatory responses (6). Furthermore, TLR2 serves a vital role in the activation of the inflammatory immune response during myocardial I/R injury by regulating pro-inflammatory cytokine induction. Cytokines such as interleukin (IL)-1 $\beta$ and IL-6 can act on cardiomyocyte membrane receptors and initiate a series of signal cascades, which in turn causes the activation of TLR2, thus leading to myocardial inflammatory damage (7). Moreover, inhibition of TLR2 with 
its ligands (e.g. peptidoglycan, Pam3CSK4) or receptor antagonists (OPN-305) has been shown to significantly decrease neutrophil infiltration, cytokine production and cardiomyocyte apoptosis $(8,9)$. Therefore, targeting TLR 2 may be a promising therapy for I/R injury.

Ischemic postconditioning (IPostC) refers to transient, repetitive $\mathrm{I} / \mathrm{R}$ in the early stage of reperfusion and has been revealed to alleviate myocardial $\mathrm{I} / \mathrm{R}$ injury in a number of animal and human models $(10,11)$. Moreover, previous studies $(12,13)$ have demonstrated that the protective effect of IPostC is closely associated with the inhibition of TLR2-induced inflammation (13); however, the underlying mechanism has not been fully elucidated. Our previous study identified that IPostC exerts a cardioprotective effect via protein kinase $\mathrm{C}$ (PKC) signaling (14), but the association between TLR2 and PKC signaling in IPostC remains unknown.

MicroRNAs (miRs) are small non-coding RNAs that serve an important role in the regulation of the natural immune response and inflammation $(15,16)$, and are involved in cardiovascular diseases $(17,18)$. miR-499, a cardiac-enriched small RNA molecule, is involved in regulating cardiac function, the pathogenesis of $\mathrm{I} / \mathrm{R}$ injury and has cardioprotective effects $(19,20)$. Furthermore, a previous study (21) demonstrated that miR-499 is involved in the anti-inflammatory and anti-apoptotic effects of IPostC.

Therefore, based on the important role of miR-499 in IPostC-induced cardioprotection, the present study investigated the relevance of the regulation of TLR2 during IPostC. A myocardial I/R rat model was established to examine the effects of IPostC on infarct size and cardiomyocyte apoptosis. Moreover, the mRNA expression level of miR-499, the serum and protein expression levels of TLR2 and PKC and inflammatory cytokines, including IL- $1 \beta$ and IL- 6 , were detected. In addition, the protein expression levels of anti- and pro-apoptotic markers, and the effects of miR-499 overexpression and inhibition on IPostC were investigated.

\section{Materials and methods}

Experimental animals. The Experimental Animal Center of Guangxi Medical University provided 90 adult male Sprague-Dawley rats $\sim 8$ weeks (weight, $240-280 \mathrm{~g}$ ). The experimental environment was maintained at a constant room temperature (temperature, $22-24^{\circ} \mathrm{C}$; relative humidity, $35-65 \%$; 12:12-h light: dark cycle) and rats were allowed ad libitum access to food and water. The experimental protocols were performed in strict accordance with the National Institutes of Health Guide for the Care and Use of Laboratory Animals and were approved by the Animal Protection and Use Committee of Guangxi Medical University.

Myocardial I/R model. Sodium pentobarbital $(2 \%, 50 \mathrm{mg} / \mathrm{kg})$ was intraperitoneally injected to anesthetize the rats. The rats were mechanically ventilated with oxygen-enriched room air using a small animal respirator. A metal syringe needle was subcutaneously inserted into all four limbs of each rat. The needle was connected to an electrocardiograph with an electric clip that recorded the cardiac electrical activity of the rat. An incision was made in the 5th intercostal space on the left sternal border to fully expose the heart. The left anterior descending coronary (LAD) artery was ligated $\sim 2 \mathrm{~mm}$ below the left atrial appendage and the angle of the articular cone with a suture with a nylon 6-0 needle. In the LAD artery, the end of the suture was passed through a small plastic tube to form a reversible snare LAD occlusion. Myocardial ischemia was induced by tightening the ligature around the plastic tube; the relaxation of the ligation caused reperfusion of the myocardial tissue. The monitoring of the changes in the ST-T segment of the electrocardiogram confirmed that ligation was successful, as indicated when the ST-T segment of the anterior region of the heart increased. Cervical dislocation of anesthetized rats was conducted at the end of reperfusion, and $3 \mathrm{ml}$ blood samples and the anterior wall of the left ventricle near the apex were collected for analysis.

Experimental groups. Rats were randomized into six groups $(\mathrm{n}=15)$ : i) Sham group, in which the ligature was passed, but not tied, and maintained for $150 \mathrm{~min}$; ii) I/R group, in which rats were subjected to $30 \mathrm{~min}$ of ischemia followed by $2 \mathrm{~h}$ of reperfusion; iii) IPostC group, in which rats underwent 3 cycles of $30 \mathrm{sec}$ of reperfusion and $30 \mathrm{sec}$ of ischemia immediately after the onset of reperfusion; iv) IPostC + negative control (NC) group, which received a miR-449 NC adeno-associated virus (AAV) vector (Hanbio Biotechnology Co., Ltd.; dose $=1 \times 10^{11}$ v.g./rat) injected into the tail vein, the operation was performed after 2 weeks of routine feeding, as in the IPostC group; v) IPostC + mimics group, which received a miR-499-overexpressing AAV vector (Hanbio Biotechnology Co., Ltd.; 1x10 11 v.g./rat) injected intravenously, with routine feeding allowed for 2 weeks, as in the IPostC group; and vi) IPostC + inhibitor group, which received miR-499 inhibitor AAV vector (Hanbio Biotechnology Co., Ltd.; dose, $1 \times 10^{11}$ v.g./rat) injected into the tail vein, followed by operation after 2 weeks, as in the IPostC group. In total, three of the 90 rats used in this study were excluded: One in the I/R group due to ventricular fibrillation; one in the IpostC $+\mathrm{NC}$ group due to cardiogenic shock during reperfusion; and one in the IPostC + inhibitors group due to viral transfection failure. The results described are for the remaining 87 rats.

$A A V$ vector transfection. The miR-499 miR-499-overexpression AAV vector (5'-GCTGTTAAGACTTGCAGTG ATGTTTAGCTCCTCTCCATGTGAACATCACAGCAAG TCTGTGCTGC-3'), miR-499 inhibitor AAV vector (5'-AAA CATCACTGCAAGTCTTAATATACAAACATCACTGCA AGTCTTAAACATCAAACATCACTGCAAGTCTTAATC TTCAAAACATCACTGCAAGTCTTAA-3') and miR-499 NC AAV vectors (Hanbio Biotechnology Co., Ltd.) were constructed. The viral vector $\left(1 \times 10^{11} \mathrm{v} . \mathrm{g} . / \mathrm{rat}\right)$ was injected into the tail vein of rats, and the expression level of miR-499 in the myocardial tissue was detected by reverse transcription-quantitative (RT-q) PCR following 2 weeks of routine feeding to confirm that the transfection was successful.

Myocardial infarction size analysis. Following reperfusion, the LAD was re-ligated and 3\% Evans blue dye at room temperature was injected into the inferior vena cava and reacted for $\sim 5$ min to identify areas of risk for myocardial 
infarction. The stained heart was removed, frozen, cut into $2-\mathrm{mm}$ sections and then incubated for $15 \mathrm{~min}$ at $37^{\circ} \mathrm{C}$ in $1 \%$ 2,3,5-triphenyltetrazolium chloride (Sigma-Aldrich; Merck $\mathrm{KGaA}$ ) to examine the size of the infarct. The infarct and risk zones were quantified using Image-Pro Plus software (version 6.0; Media Cybernetics, Inc.), as previously described (14). The infarct size was expressed as a percentage of the risk area, and the risk area was calculated as the percentage of the left ventricle.

TUNEL staining. The histochemical detection of apoptotic cells was performed as previously reported (14). Apoptotic cells were identified using a TUNEL detection kit (cat. no. 116848179; Roche Diagnostics GmbH) according to the manufacturer's protocol. The dewaxed myocardial tissue sections were immersed in $3 \%$ hydrogen peroxide in methanol for $10 \mathrm{~min}$ at room temperature, and washed 3 times with PBS; then the tissue sections and proteinase K working solution (cat. no. G1205; WuHan Servicebio Technology Co., Ltd.) incubated at $37 \mathrm{C}$ for $30 \mathrm{~min}$, which the tissue sections and TdT reaction mixture are incubated at $37 \mathrm{C}$ for $2 \mathrm{~h}$. After washing 3 times with PBS, counterstain the nuclei with DAPI solution (cat. no. D1306; 1:1,000; Thermo Fisher Scientific, Inc.) at room temperature for $5 \mathrm{~min}$, and use $50 \mu 1$ of anti-fade mounting medium (cat. no. G1401; WuHan Servicebio Technology Co., Ltd.) TUNEL-positive cells were observed in five randomly selected areas using a fluorescence microscope (magnification, $\mathrm{x} 200$ ). The apoptotic index was expressed as the number of TUNEL-positive cells/total number of cardiomyocytes $\mathrm{x} 100 \%$.

Levels of TLR2, PKC, IL- $1 \beta$ and IL- 6 in serum. Prior to the removal of the heart, 5-ml blood samples were collected and centrifuged at $4 \mathrm{C}, 2,000 \mathrm{x}$ for $10 \mathrm{~min}$, and the supernatant was stored in a refrigerator at $-80 \mathrm{C}$. The serum levels of TLR2, PKC, IL-1 $\beta$ and IL-6 were assessed with an automatic analyzer 7600 (Hitachi Ltd.) using rat TLR2 ELISA kit (cat. no. 0663R1; Enzyme immunoassay Co.), rat PKC ELISA kit (cat. no. 0684R1; Enzyme immunoassay Co.), rat IL-1 $\beta$ ELISA kit (cat. no. 0047R1; Enzyme immunoassay Co.) and rat IL-6 ELISA kit (cat. no. 0190R1; Enzyme immunoassay Co.). The experiment was repeated four times.

RNA extraction and RT-qPCR. Total RNA was extracted using TRIzol ${ }^{\circledR}$ reagent (Invitrogen; Thermo Fisher Scientific, Inc.) according to the manufacturer's instructions. First-strand cDNA was synthesized from $1 \mu \mathrm{g}$ total RNA using a RevertAid First Strand cDNA Synthesis kit (Thermo Fisher Scientific, Inc.) and reverse transcribed using the following temperature protocol: $37 \mathrm{C}$ for $15 \mathrm{~min}, 85 \mathrm{C}$ for $5 \mathrm{sec}$ and stored at 4C. RT-qPCR was performed using 2X SYBR Green qPCR ProMix (EnzyValley) in an ABI 7300 RT PCR system (Applied Biosystems; Thermo Fisher Scientific, Inc.). The reaction mixtures were incubated in a 96-well plate and the RT-qPCR thermal cycling conditions were as follows: Initial denaturation at $95^{\circ} \mathrm{C}$ for $2 \mathrm{~min}$, followed by 40 cycles of $95^{\circ} \mathrm{C}$ for $5 \mathrm{sec}$ and final extension at 40 cycles of $60^{\circ} \mathrm{C}$ for $30 \mathrm{sec}$. RNU6B was used as the reference control. Primer sequences are presented in Table I. The relative expression level of miR-499 was determined using the $2^{-\Delta \Delta \mathrm{Cq}}$ method (22).
Western blotting. Western blotting was performed as described previously (14). Freshly frozen myocardial tissue samples were homogenized in RIPA buffer (Beyotime Institute of Biotechnology). The protein concentration was determined with a bicinchoninic acid assay. Equal amounts $(30 \mu \mathrm{g})$ of proteins were separated by $12 \%$ SDS-PAGE and were transferred to nitrocellulose membranes that were blocked with $5 \%$ non-fat milk for $1 \mathrm{~h}$ at room temperature in TBST $(150 \mathrm{mM} \mathrm{NaCl}, 20 \mathrm{mM}$ Tris- $\mathrm{HCl}$ and $0.1 \%$ Tween $20 ; \mathrm{pH}$ 7.4). All antibodies were acquired from Cell Signaling Technology, Inc. Membranes were incubated with TLR2 rabbit monoclonal antibodies (cat. no. 12276S; 1:1,000), PKC rabbit monoclonal antibodies (cat. no. 38938S; 1:1,000.), IL-1ßrabbit monoclonal antibodies (cat. no. 12703S; 1:1,000.), IL-6 rabbit monoclonal antibodies (cat. no. 12912S; 1:1,000.), Bcl-2 rabbit monoclonal antibodies (cat. no. 3498S; 1:1,000) and Bax rabbit monoclonal antibodies (cat. no. 5023S; 1:1,000) overnight at 4C. After washing 3 times with TBST, the membranes were exposed to peroxidase-conjugated goat anti-rabbit $\operatorname{IgG}(\mathrm{H}+\mathrm{L})$ antibodies (cat. no. 14708S; 1:3,000) for $1 \mathrm{~h}$ at room temperature. Finally, using Chemiluminescence substrate (Pierce; Thermo Fisher Scientific, Inc.) and exposed on radiographic film. The results were quantitatively analyzed using ImageJ v1.8.0 software (National Institutes of Health).

Statistical analysis. Data are presented as the mean \pm SD. One-way ANOVA was performed using Tukey's multiple comparison test. $\mathrm{P}<0.05$ was considered to indicate a statistically significant difference. All statistical analyses were performed using SPSS 23.0 (SPSS, Inc.).

\section{Results}

Effect of IPostC on the expression levels of inflammatory cytokines. The inhibition of inflammatory cytokine activation serves an important role in mitigating myocardial I/R injury (23). In order to determine whether IPostC exerts cardioprotective effects by inhibiting the activation of inflammatory cytokines, the present study examined the serum and the protein expression levels of TLR2, IL-1 $\beta$ and IL-6. It was identified that, when comparing the I/R group with the IPostC group, the serum and protein expression levels of TLR2 (serum: $23.1 \pm 3.3$ vs. $28.4 \pm 3.9$; protein: $0.57 \pm 0.03$ vs. $0.80 \pm 0.04$, respectively; $\mathrm{P}<0.05$ ), IL-1 $\beta$ (serum: $51.9 \pm 5.0$ vs. $60.6 \pm 5.8$; protein: $0.51 \pm 0.03$ vs. $0.75 \pm 0.03$, respectively; $\mathrm{P}<0.05$ ) and IL-6 (serum: $108.7 \pm 8.9$ vs. $156.9 \pm 10.5$; protein: $0.46 \pm 0.03$ vs. $0.70 \pm 0.03$, respectively; $\mathrm{P}<0.05)$ were significantly decreased in the IPostC group (Table II; Fig. 1). Therefore, the results indicated that IPostC may alleviate myocardial I/R damage by inhibiting the activation of inflammatory cytokines.

Effect of IPostC on the expression of PKC. To determine the signal transduction mechanism via which IPostC may serve a role in cardioprotection, the serum and the protein expression levels of PKC were measured. It was identified that the serum and the protein expression levels of PKC were significantly decreased in the IPostC group compared with the I/R group (serum: $720.1 \pm 24.9$ vs. $1,036.5 \pm 30.1$; protein: $0.57 \pm 0.03$ vs. $0.80 \pm 0.04$, respectively; $\mathrm{P}<0.05$; Table II; Fig. 2 ). Thus, it was 
Table I. Sequences of primers used for reverse transcription-quantitative PCR analyses.

\begin{tabular}{lll}
\hline Genes & Primers & \multicolumn{1}{c}{ Sequences } \\
\hline U6 & Forward & 5'-CTCGCTTCGGCAGCACA-3' \\
miR-499 & Reverse & 5'-AACGCTTCACGAATTTGCGT-3' \\
& Forward & 5'-TTAAGACTTGCAGTGATGTTT-3' \\
miRT Random Primer & Reverse & 5'-CAGTGCAGGGTCCGAGGTAT-3' \\
& & 5'-GTCGTATCCAGTGCAGGGTCCGAGGTATTCGCAC \\
& & TGGATACGACNNNNN-3'
\end{tabular}

miR, microRNA.

Table II. Effects of IPostC with the miR-499 mimic, inhibitor and negative control AAV vectors on the levels of TLR2, PKC, IL-1 $\beta$ and IL-6 in the serum following myocardial I/R.

\begin{tabular}{lcccc}
\hline Groups & TLR2,pg/ml & PKC, pg/ml & IL-1 $\beta, \mathrm{pg} / \mathrm{ml}$ & IL-6,pg/ml \\
\hline Sham & $16.7 \pm 2.8$ & $320.4 \pm 17.2$ & $35.5 \pm 4.2$ & $68.4 \pm 7.3$ \\
I/R & $28.4 \pm 3.9^{\mathrm{a}}$ & $1036.5 \pm 30.1^{\mathrm{a}}$ & $60.6 \pm 5.8^{\mathrm{a}}$ & $156.9 \pm 10.5^{\mathrm{a}}$ \\
IPostC & $23.1 \pm 3.3^{\mathrm{b}}$ & $720.1 \pm 24.9^{\mathrm{b}}$ & $51.9 \pm 5.0^{\mathrm{b}}$ & $108.7 \pm 8.9^{\mathrm{b}}$ \\
IPostC + NC & $22.4 \pm 3.2^{\mathrm{b}}$ & $717.2 \pm 26.0$ & $52.1 \pm 3.0$ & $107.9 \pm 8.0$ \\
IPostC + inhibitors & $32.8 \pm 5.5^{\mathrm{c}}$ & $987.7 \pm 28.7^{\mathrm{c}}$ & $68.5 \pm 3.1^{\mathrm{c}}$ & $178.5 \pm 7.8^{\mathrm{c}}$ \\
IPostC + mimics & $13.6 \pm 2.9^{\mathrm{c}}$ & $505.2 \pm 19.7^{\mathrm{c}}$ & $37.0 \pm 1.8^{\mathrm{c}}$ & $77.1 \pm 4.9^{\mathrm{c}}$ \\
\hline
\end{tabular}

Data are presented as the mean $\pm \mathrm{SD}$. ${ }^{\mathrm{a}} \mathrm{P}<0.05$ vs. sham group. ${ }^{\mathrm{b}} \mathrm{P}<0.05$ vs. I/R group. ${ }^{\mathrm{C}} \mathrm{P}<0.05 \mathrm{vs}$. IPostC group. $\mathrm{n}=8$ per group. IL, interleukin; TLR, Toll-like receptor; PKC, protein kinase C; NC, negative control; IPostC, ischemic postconditioning; IR, ischemia/reperfusion; miR, microRNA.
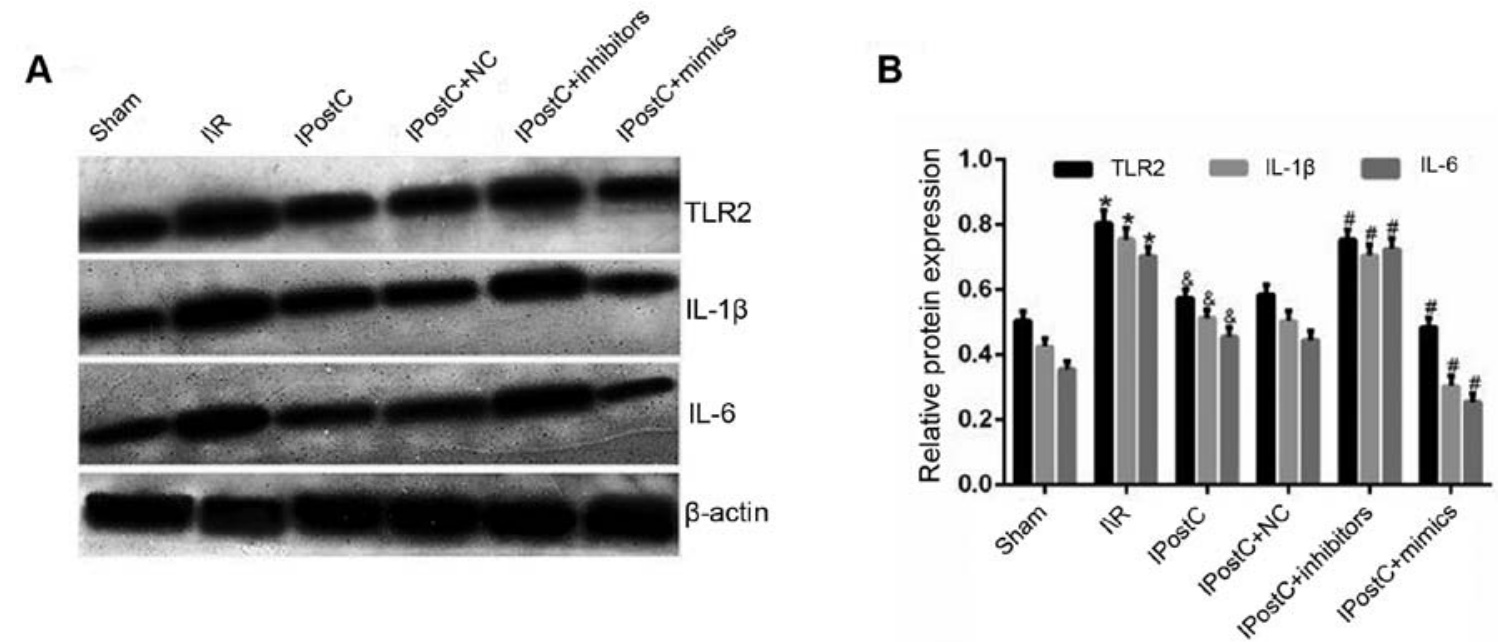

Figure 1. Effect of IPostC on the expression levels of inflammatory cytokines. (A) Effects of IPostC with or without treatment with the miR-499-5p mimic, inhibitor and NC AAV vectors on TLR2, IL-1 $\beta$ and IL-6 protein expression levels following myocardial I/R. (B) Quantitative analysis of TLR2, IL-1 $\beta$ and IL-6 protein expression levels. Data are presented as the mean $\pm \mathrm{SD}$. ${ }^{*} \mathrm{P}<0.05$ vs. sham group. ${ }^{~} \mathrm{P}<0.05 \mathrm{vs}$. I/R group. ${ }^{\#} \mathrm{P}<0.05$ vs. IPostC group. $\mathrm{n}=6$ per group. NC, negative control; miR, microRNA; TLR, Toll-like receptor; IL, interleukin; IPostC, ischemic postconditioning; IR, ischemia/reperfusion; AAV, adeno-associated virus.

hypothesized that IPostC may have a cardioprotective role by inhibiting the PKC signal transduction pathway.

Effect of IPostC on the protein expression levels of Bcl-2 and Bax. The results demonstrated that the IPostC group exhibited an increased expression of Bcl-2 (0.63 \pm 0.03 vs. $0.25 \pm 0.02 ; \mathrm{P}<0.05)$ and a decreased expression level of Bax $(0.49 \pm 0.02$ vs. $0.78 \pm 0.03 ; \mathrm{P}<0.05)$ in the myocardium compared with the I/R group (Fig. 3). Moreover, these results suggested that IPostC protected the heart from I/R-induced 
A

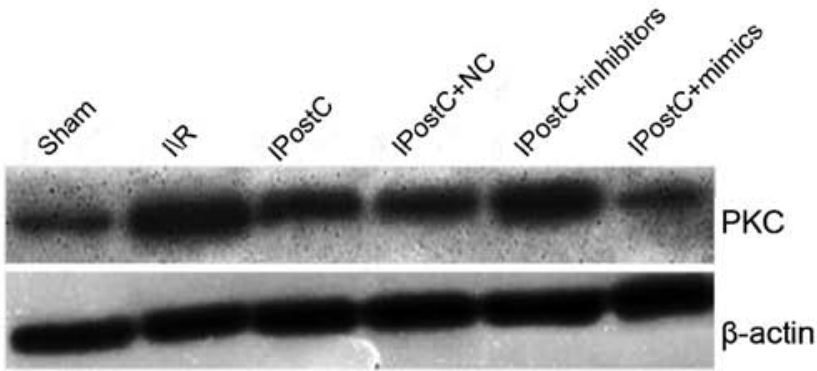

B

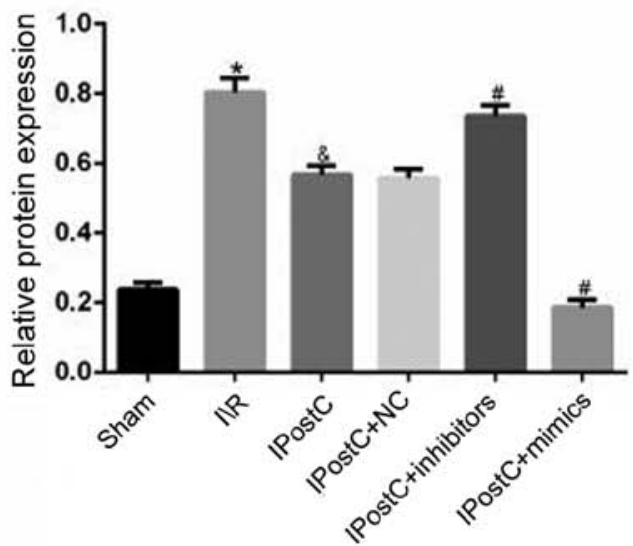

Figure 2. Effect of miR-499 on the expression level of PKC. (A) Effects of IPostC with or without treatment with the miR-499-5p mimic, inhibitor and NC AAV vectors on PKC protein expression following myocardial I/R. (B) Quantitative analysis of PKC protein expression. Data are presented as the mean \pm SD. ${ }^{*} \mathrm{P}<0.05$ vs. sham group. ${ }^{\&} \mathrm{P}<0.05$ vs. I/R group. ${ }^{\text {" }} \mathrm{P}<0.05$ vs. IPostC group. $\mathrm{n}=6$ per group. NC, negative control; miR, microRNA; IPostC, ischemic postconditioning; IR, ischemia/reperfusion; AAV, adeno-associated virus; PCK, protein kinase C.
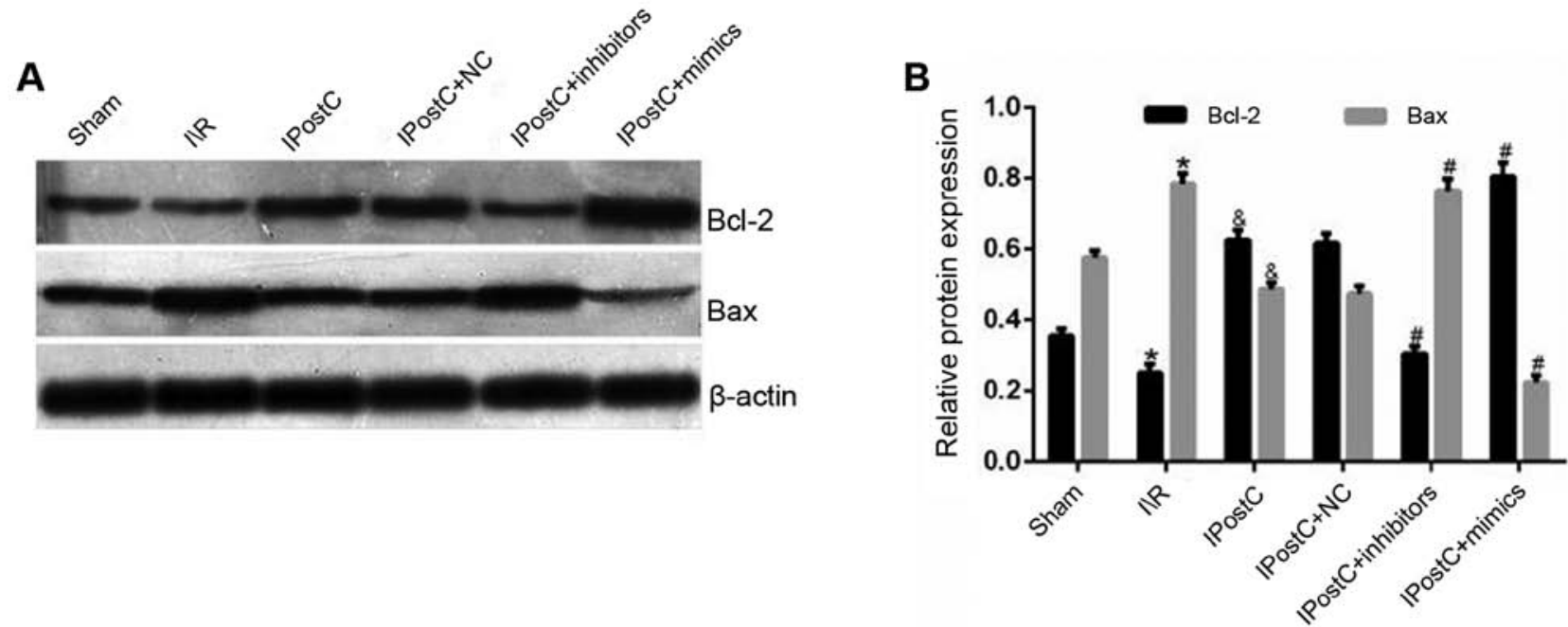

Figure 3. Effect of IPostC on the protein expression levels of Bcl-2 and Bax. (A) Effects of IPostC with or without treatment with the miR-499-5p mimic, inhibitor and NC AAV vectors on Bcl-2 and Bax protein expression levels following myocardial I/R. (B) Quantitative analysis of Bcl-2 and Bax protein expression levels. Data are presented as the mean $\pm \mathrm{SD}$. ${ }^{*} \mathrm{P}<0.05$ vs. sham group. ${ }^{\circledR} \mathrm{P}<0.05$ vs. $\mathrm{I} / \mathrm{R}$ group. ${ }^{~} \mathrm{P}<0.05$ vs. IPostC group. $\mathrm{n}=6$ per group. $\mathrm{NC}$, negative control; miR, microRNA; IPostC, ischemic postconditioning; IR, ischemia/reperfusion; AAV, adeno-associated virus.

apoptosis by increasing Bcl-2 and decreasing Bax protein expression levels.

Effect of IPostC on the mRNA expression level of miR-499. To determine whether miR-499 was associated with the cardioprotective effects of IPostC, the mRNA expression level of miR-499 was examined in myocardial tissue. It was identified that the mRNA expression level of miR-499 in the IPostC group was significantly increased compared with the I/R group (1.27 \pm 0.09 vs. $0.65 \pm 0.13 ; \mathrm{P}<0.05$; Fig. 4 ).

Expression levels of miR-499 in the myocardium following viral transfection. In order to further investigate the effect of miR-499 on the cardioprotective mechanism of IPostC, the rat myocardium was transfected with miR-499 mimic, inhibitor and NCAAV vectors and then the mRNA expression of miR-499 was measured. It was identified that there was no significant difference in mRNA expression level of miR-499 between the IPostC $+\mathrm{NC}$ and IPostC groups $(1.33 \pm 0.09$ vs. $1.27 \pm 0.09$; Fig. 4). However, compared with the miR-499 expression levels in the IPostC group, the level in the IPostC + inhibitors group was significantly decreased $(0.59 \pm 0.12$ vs. $1.27 \pm 0.09$; $\mathrm{P}<0.05)$, while that in the IPostC + mimics group was significantly increased $(1.80 \pm 0.16$ vs. $1.27 \pm 0.09 ; \mathrm{P}<0.05)$. Therefore, these experimental results identified that the transfection of the miR-499 virus was successful.

Effect of miR-499 on the expression levels of inflammatory factors. The results demonstrated that the protein expression levels of TLR2 $(0.58 \pm 0.03$ vs. $0.57 \pm 0.03 ; \mathrm{P}>0.05)$, IL-1 $\beta(0.50 \pm 0.03$ vs. $0.51 \pm 0.03 ; \mathrm{P}>0.05)$ and IL-6 $(0.44 \pm 0.03$ vs. $0.46 \pm 0.03 ; \mathrm{P}>0.05)$ were not significantly different between the IPostC + NC and IPostC groups (Fig. 1). Moreover, compared with the IPostC group, the expression levels of TLR2 


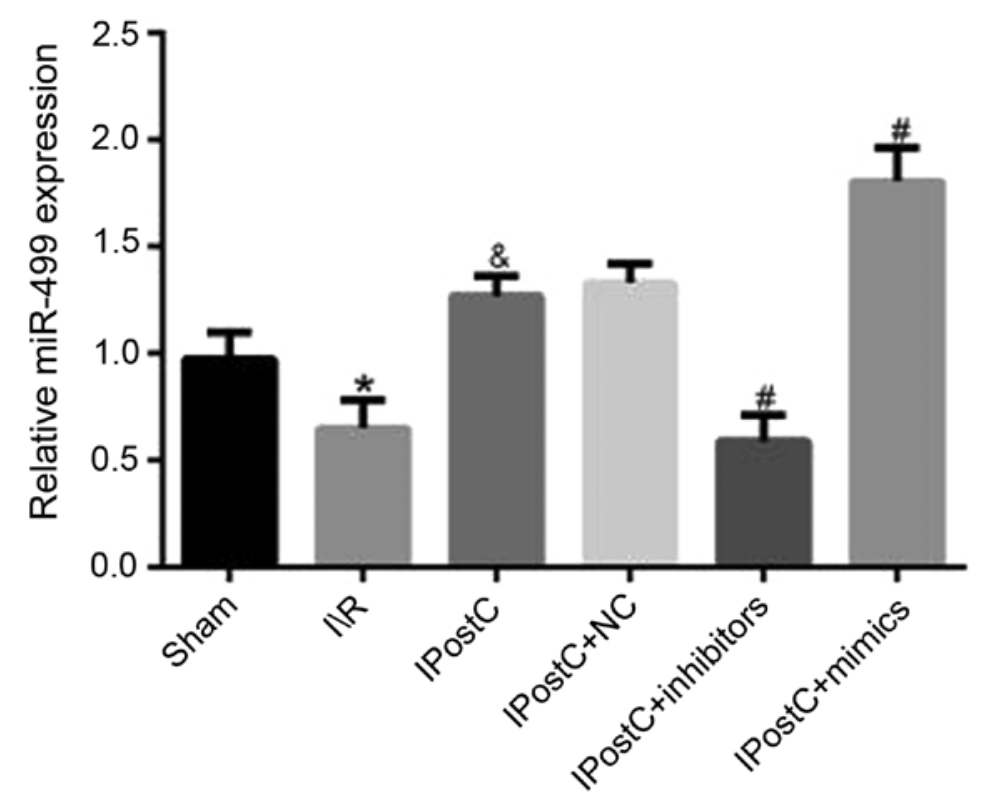

Figure 4. Effect of IPostC on the mRNA expression level of miR-499. Effects of IPostC with or without treatment with the miR-499-5p mimic, inhibitor and NC AAV vectors on miR-499 mRNA expression level following myocardial I/R. Data are presented as the mean $\pm \mathrm{SD}$. ${ }^{*} \mathrm{P}<0.05$ vs. sham group. ${ }^{\circledR} \mathrm{P}<0.05$ vs. I/R group. " $\mathrm{P}<0.05$ vs. IPostC group. $\mathrm{n}=6$ per group. NC, negative control; miR, microRNA; IPostC, ischemic postconditioning; IR, ischemia/reperfusion; AAV, adeno-associated virus.

$(0.75 \pm 0.03$ in IPostC + inhibitors vs. $0.57 \pm 0.03$ in IPostC; $\mathrm{P}<0.05), \mathrm{IL}-1 \beta(0.71 \pm 0.03$ in IPostC + inhibitors vs. $0.51 \pm 0.03$ in IPostC; $\mathrm{P}<0.05)$ and IL-6 $(0.73 \pm 0.03$ in IPostC + inhibitors vs. $0.46 \pm 0.03$ in IPostC; $\mathrm{P}<0.05)$ were significantly increased in the IPostC + inhibitors group, while the expression levels of TLR2 $(0.48 \pm 0.03$ in IPostC + mimics vs. $0.57 \pm 0.03$ in IPostC; $\mathrm{P}<0.05)$, IL-1 $\beta(0.30 \pm 0.03$ in IPostC + mimics vs. $0.51 \pm 0.03$ in IPostC; $\mathrm{P}<0.05)$ and IL-6 $(0.25 \pm 0.02$ in IPostC + mimics vs. $0.46 \pm 0.03$ in IPostC; $P<0.05)$ were significantly decreased in the IPostC + mimics group. Collectively, these results suggested that the upregulation of miR-499 can exert cardioprotective effects by inhibiting the expression level of inflammatory cytokines.

Effect of miR-499 on the expression level of PKC. It was identified that there was no significant difference in PKC expression levels between the IPostC $+\mathrm{NC}$ group and IPostC groups (Table II; Fig. 2). Furthermore, the serum and the protein expression levels of PKC were significantly increased in the IPostC + inhibitor group $(987.7 \pm 28.7$ vs. $720.1 \pm 24.9$ and $0.74 \pm 0.03$ vs. $0.57 \pm 0.03$, respectively; $\mathrm{P}<0.05)$ and significantly decreased in the IPostC + mimics group (505.2 \pm 19.7 vs. $720.1 \pm 24.9$ and $0.19 \pm 0.02$ vs. $0.57 \pm 0.03$, respectively; $\mathrm{P}<0.05)$ compared with those in the IPostC group. Therefore, it was speculated that the upregulation of miR-499 may exert anti-inflammatory effects by inhibiting the PKC signal transduction pathway.

Effect of miR-499 on the expression level of circulating inflammatory cytokines. To further assess the effect of miR-499 on the systemic inflammatory status of IPostC, the rat myocardium was transfected with miR-499 mimic, inhibitor and NC AAV vectors and the serum levels of inflammatory cytokines were measured. The results demonstrated that the serum levels of TLR2, IL-1 $\beta$ and IL- 6 were not significantly different between the IPostC $+\mathrm{NC}$ and IPostC groups (Table II). In comparison with the IPostC $+\mathrm{NC}$ and IPostC groups, the serum levels of TLR2, IL-1 $\beta$ and IL- 6 were significantly increased in the IPostC + inhibitors group and were all significantly decreased in the IPostC + mimics group.

Effect of miR-499 on cardiomyocyte apoptosis and myocardial infarct size. To assess whether miR-499 serves a role in the cardioprotective effects of IPostC, the apoptotic index of cardiomyocytes (Fig. 5) and myocardial infarct size (Fig. 6) were examined. Relative to the I/R group, the IPostC group exhibited a significantly decreased myocardial apoptotic index $(29.46 \pm 2.03 \%$ vs. $42.64 \pm 2.27 \%$; $\mathrm{P}<0.05)$ and significantly decreased myocardial infarct size $(48.53 \pm 2.49 \%$ vs. $66.52 \pm 3.1 \%$; $\mathrm{P}<0.05)$. Furthermore, the cardiomyocyte apoptotic index $(28.00 \pm 1.54 \%$ vs. $29.46 \pm 2.03 \%$; $>0.05)$ and myocardial infarct size $(47.53 \pm 2.47 \%$ vs. $48.53 \pm 2.49 \%$; $\mathrm{P}>0.05)$ were not significantly different between the IPostC $+\mathrm{NC}$ group and the IPostC group. However, compared with those in the IPostC group, the myocardial apoptotic index $(37.45 \pm 1.73 \%$ vs. $29.46 \pm 2.03 \% ; \mathrm{P}<0.05)$ and myocardial infarct size $(60.18 \pm 2.81 \%$ vs. $48.53 \pm 2.49 \%$; $\mathrm{P}<0.05)$ were significantly increased in the IPostC + inhibitors group, but significantly decreased in the IPostC + mimics group (cardiomyocyte apoptotic index, $18.13 \pm 1.83 \%$ vs. $29.46 \pm 2.03 \%$; $\mathrm{P}<0.05$; myocardial infarct size, $26.73 \pm 2.45 \%$ vs. $48.53 \pm 2.49 \% ; \mathrm{P}<0.05)$. Collectively, the results suggested that the upregulation of miR-499 could effectively alleviate myocardial I/R damage and may be involved in the ischemic myocardial protection mechanism of IPostC.

Effect of miR-499 on the protein expression levels of Bcl-2 and Bax. It was found that the protein expression levels of Bcl-2 and Bax were not significantly different between the IPostC + NC and IPostC groups (Fig. 3). In comparison with the IPostC group, the IPostC + mimics group exhibited an 


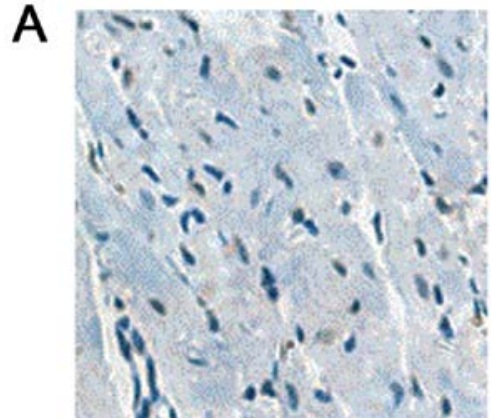

Sham group

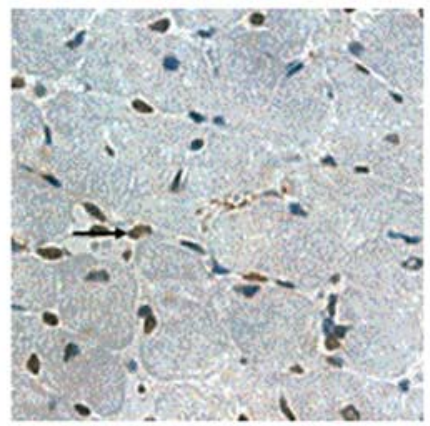

IPostC+NC group

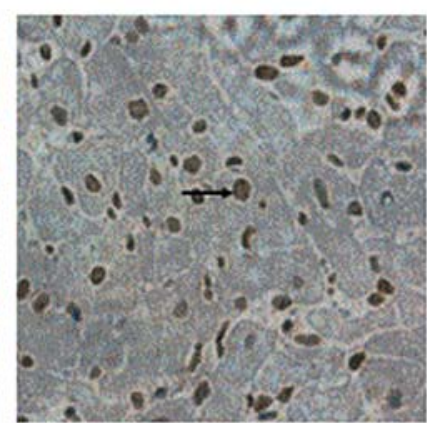

I/R group

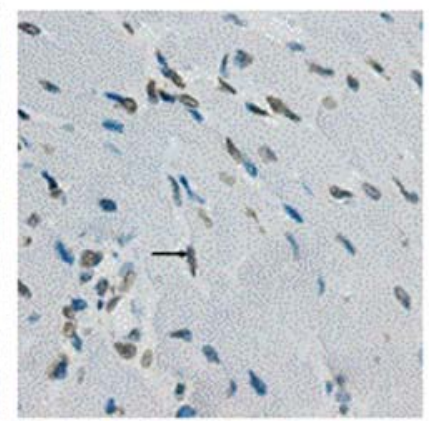

IPostC+inhibitors group

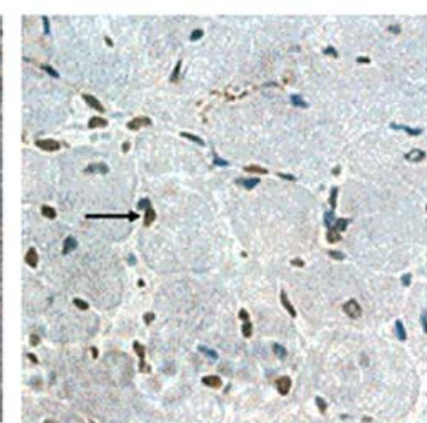

IPostC group

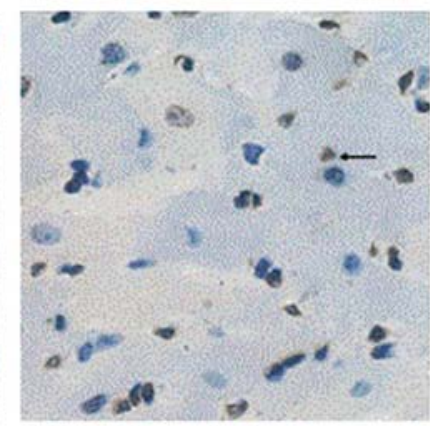

IPostC+mimics group

B

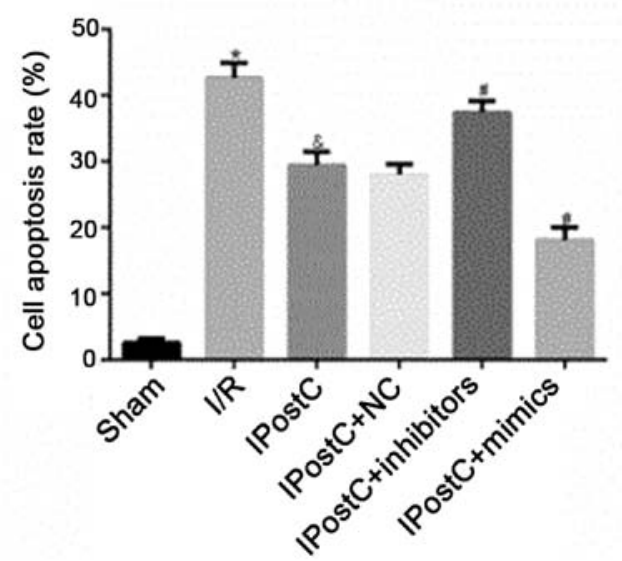

Figure 5. Effect of miR-499 on cardiomyocyte apoptosis. (A) Effects of IPostC with or without treatment with the miR-499-5p mimic, inhibitor and NC AAV vectors on apoptosis following myocardial I/R. Magnification, x200. (B) Quantitative analysis of cell apoptotic rate, data are presented as the mean \pm SD. ${ }^{*} \mathrm{P}<0.05$ vs. sham group. ${ }^{\circledR} \mathrm{P}<0.05$ vs. I/R group. ${ }^{\sharp} \mathrm{P}<0.05$ vs. IPostC group. $\mathrm{n}=4$ per group. NC, negative control; miR, microRNA; IPostC, ischemic postconditioning; IR, ischemia/reperfusion; AAV, adeno-associated virus.

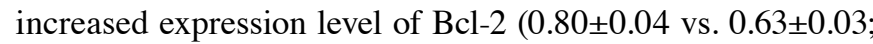
$\mathrm{P}<0.05)$ and a decreased expression level of $\mathrm{Bax}(0.22 \pm 0.02 \mathrm{vs}$. $0.49 \pm 0.02 ; \mathrm{P}<0.05)$ in the myocardium; however, these effects were reversed by miR-499 inhibitors.

\section{Discussion}

The present results suggested that IPostC significantly increased miR-499 mRNA expression level, but decreased the I/R-induced infarct size, cardiomyocyte apoptosis and the release of the inflammatory mediators, including TLR2, PKC, IL-1 $\beta$ and IL-6. To the best of our knowledge, the present study was the first to demonstrate that miR-499 exerted an ischemic cardioprotective effect in IPostC, possibly by inhibiting TLR2 and PKC signals, thereby decreasing cardiomyocyte necrosis and apoptosis via the induction of the anti-apoptotic protein Bcl-2 and suppression of the pro-apoptotic Bax. The present results identified a potentially novel mechanism of IPostC-induced protection.

The pattern recognition receptor TLR2 is an emerging therapeutic target in $\mathrm{I} / \mathrm{R}$ injury and cardioprotection. Sakata et al (24) revealed that TLR2 is released from mouse myocardium and mediates subsequent injury during I/R. Furthermore, TLR2 upregulation is observed in the monocytes of patients with acute myocardial infarction, which contributes to the severity of myocardial damage (25). By contrast, the application of TLR2 monoclonal antibody has been shown to result in significant decreases in myocardial infarction size and local and systemic inflammatory cells (26). It has also been shown that preconditioning attenuates myocardial injury by inhibiting the activation of TLR2 (27-29). However, whether IPostC has a similar effect on the inhibition of TLR 2 signaling 
A

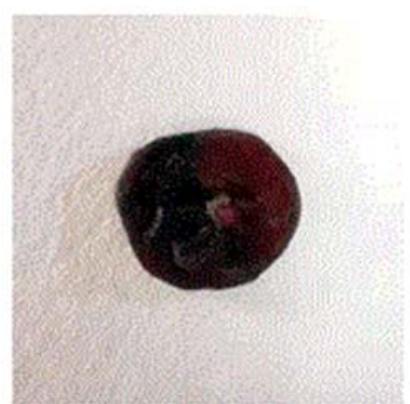

Sham group

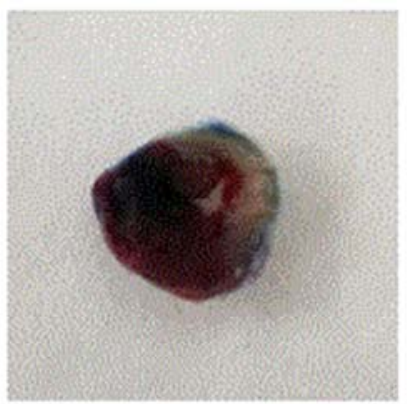

IPostC+NC group

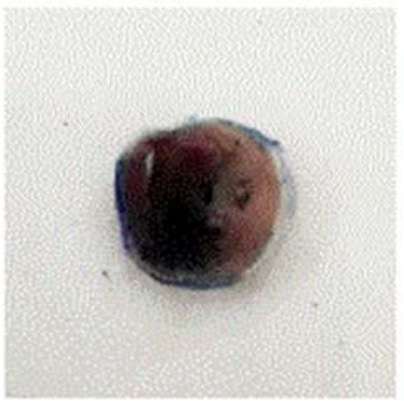

I/R group

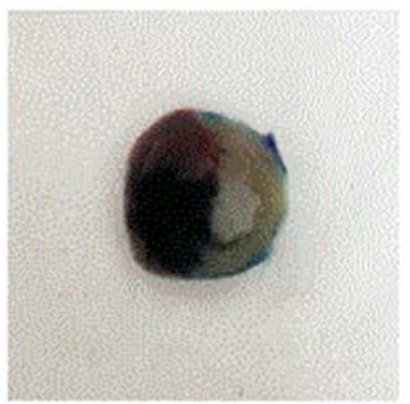

IPostC+inhibitors group

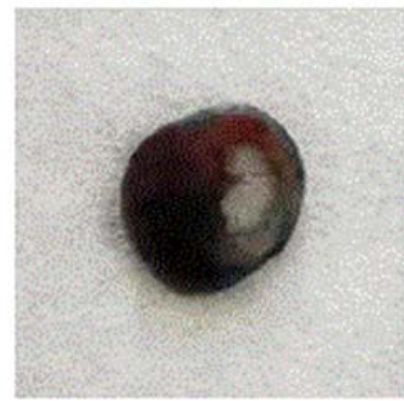

IPostC group

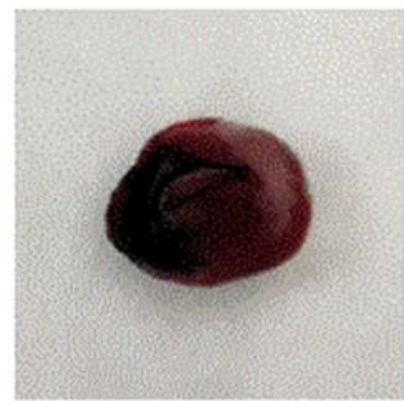

IPostC+mimics group

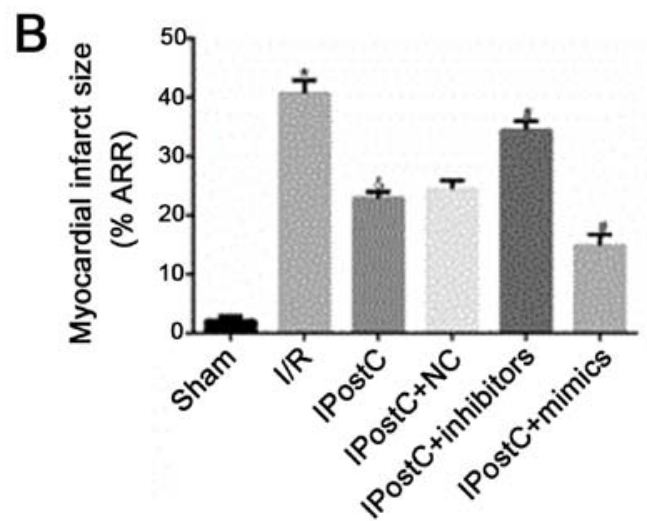

Figure 6. Effect of miR-499 on myocardial infarct size. (A) Effects of IPostC with or without treatment with the miR-499 mimic, inhibitor and NC AAV vectors on myocardial infarct size following myocardial I/R in myocardial tissue. Magnification, $\mathrm{x} 40$. (B) Quantitative analysis of myocardial infarct size, data are

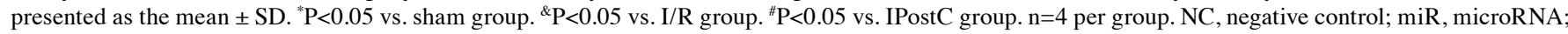
IPostC, ischemic postconditioning; IR, ischemia/reperfusion; AAV, adeno-associated virus; MI/ARR, myocardial infarction/risk area.

remains unknown. In the present study, low local and circulating levels of TLR2 were identified in the IPostC group and significantly increased levels in the I/R group, indicating that IPostC-induced cardioprotection may be closely associated with the inhibition of TLR2.

The interaction between TLR 2 and inflammatory cytokines is an important component of myocardial I/R injury (30). As a potent inflammatory mediator, activated TLR 2 promotes the accumulation of inflammatory cells and the release of inflammatory cytokines, such as IL-1 $\beta$ and IL-6, from necrotic cardiomyocytes, triggering a wide range of inflammation (31). Moreover, the concentrations of these cytokines during I/R partially reflect the severity of myocardial damage $(32,33)$. Furthermore, treatment strategies that inhibit these cytokines have been shown to be effective for reducing infarct size and cardiomyocyte apoptosis $(34,35)$. Consistent with previous studies, the present results indicated that $30-\mathrm{min}$ ischemia followed by $120-\mathrm{min}$ reperfusion significantly increased myocardial infarct size, cardiomyocyte apoptosis and the release of IL- $1 \beta$ and IL- 6 , while IPostC prevented these effects. Therefore, the results suggested that the beneficial effect of IPostC may be due to the inhibition of the inflammatory cytokines IL-1 $\beta$ and IL-6.

The PKC family is a class of signaling proteins that are often associated with the regulation of I/R (36). Moreover, PKC may mediate I/R-induced cardiomyocyte apoptosis via interactions with the mitochondria (37). PKC is also an important component of the TLR2 signal transduction pathway (38). Park et al (39) showed that TLR2 can mediate inflammatory responses by adjusting downstream PKC signaling. However, while a previous study (40) has shown that PKC signaling is linked to TLR2, to the best of our knowledge, no previous study has investigated their association in IPostC. Therefore, the aim of the present study was to determine whether TLR2 and PKC signaling pathways were involved in I/R-induced injury and IPostC-mediated cardioprotection. The present results identified 
high expression levels of TLR2 and PKC in serum and heart in the I/R group, but significantly decreased expression levels in the IPostC group, suggesting that inhibition of PKC signaling in response to TLR2 inhibition may be associated with the protective effects of IPostC. Collectively, the present study identified a potentially novel protective mechanism of IPostC.

Previous studies have shown that miR-499 prevents myocardial I/R injury. miR-499 is a small non-coding RNA that regulates the expression levels of numerous target genes in heart disease, particularly in acute myocardial infarction $(41,42)$. Qin et al $(43)$ revealed that the expression level of miR-499 in the left ventricular myocardium is negatively correlated with troponin $\mathrm{T}$ and creatine kinase-muscle/brain expression levels in a canine model of I/R. It has also been shown that miR-499 is upregulated in IPostC, and exerts anti-apoptotic and anti-inflammatory effects on the ischemic myocardium (21). However, whether the mechanisms underlying the cardioprotective effects of IPostC involve miR-499 is not fully understood. The present study examined whether the TLR2 and PKC signaling pathways were involved in these cardioprotective effects by increasing and decreasing the expression of miR-499 using miR-499 mimics and inhibitors. It was demonstrated that miR-499, which was highly expressed in the IPostC group, inhibits TLR2 and PKC signaling and the subsequent inflammatory cascade in I/R. In addition, it was identified that miR-499 inhibitors significantly increased the expression levels of TLR2 and PKC and the proinflammatory cytokines, IL-1 $\beta$ and IL-6. By contrast, the overexpression of miR-499 significantly reversed these trends. To the best of our knowledge, the present study was the first to identify the interaction between miR-499 and TLR2 in IPostC, which suggested a possible mechanism of the anti-inflammatory effect of IPostC. However, the underlying mechanism via which miR-499 inhibits TLR2 expression remains unknown.

In conclusion, the present results suggested that miR-499 may be involved in IPostC cardioprotection, possibly by inhibiting local and systemic TLR2 activation, inflammation and PKC signaling. Therefore, the present study provided mechanistic evidence to support the development of potential therapeutic targets for providing IPostC-mediated cardioprotection against I/R injury.

\section{Acknowledgements}

The authors would like to thank Professor Zhi-Yu Zeng and Dr Hong Wen (both Department of Cardiology, First Affiliated Hospital, Guangxi Medical University, Nanning, China) for their collaboration.

\section{Funding}

The present study was supported by grants from the National Natural Science Foundation of China (grant. no. 81560068) and the Natural Science Foundation of Guangxi Province (grant. no. 2015GXNSFAA139198).

\section{Availability of data and materials}

All data generated or analyzed during this study are included in this published article.

\section{Authors' contributions}

RT contributed to study conception and design, manuscript revision and obtained funding. $\mathrm{XZ}$ contributed to study design, data collection and analysis, writing of the manuscript, and general feedback on the article. $\mathrm{ZH}$ contributed to data interpretation and manuscript revision. QL contributed to data acquisition and analysis. GZ contributed to data analysis and interpretation. JM contributed to data analysis and writing of the manuscript. DW contributed to data analysis and the writing of the manuscript. All authors read and approved the final manuscript.

\section{Ethics approval and consent to participate}

The experimental protocols were performed in strict accordance with the National Institutes of Health Guide for the Care and Use of Laboratory Animals and were approved by the Animal Protection and Use Committee of Guangxi Medical University.

\section{Patient consent for publication}

Not applicable.

\section{Competing interests}

The authors declare that they have no competing interests.

\section{References}

1. Turer AT and Hill JA: Pathogenesis of myocardial ischemia-reperfusion injury and rationale for therapy. Am J Cardiol 106: 360-368, 2010

2. Hausenloy DJ, Botker HE, Engstrom T, Erlinge D, Heusch G, Ibanez B, Kloner RA, Ovize M, Yellon DM and Garcia-Dorado D: Targeting reperfusion injury in patients with ST-segment elevation myocardial infarction: Trials and tribulations. Eur Heart J 38: 935-941, 2017.

3. Yang Y, Yang J, Liu XW, Ding JW, Li S, Guo X, Yang CJ, Fan ZX, Wang HB, Li Q, et al: Down-Regulation of miR-327 alleviates ischemia/reperfusion-induced myocardial damage by targeting RP105. Cell Physiol Biochem 49: 1049-1063, 2018.

4. Pope MR and Fleming SD: TLR2 modulates antibodies required for intestinal ischemia/reperfusion-induced damage and inflammation. J Immunol 194: 1190-1198, 2015.

5. Arslan F, Keogh B, Mcguirk P and Parker AE: TLR2 and TLR4 in ischemia reperfusion injury. Mediators Inflamm 2010: 704202, 2010.

6. Chen E, Bakr MM, Firth N and Love RM: Inflammatory cell expression of Toll-like receptor-2 (TLR2) within refractory periapical granuloma. F1000Res 7: 1819, 2018.

7. Ward JR, Wilson HL, Francis SE, Crossman DC and Sabroe I: Translational mini-review series on immunology of vascular disease: Inflammation, infections and Toll-like receptors in cardiovascular disease. Clin Exp Immunol 156: 386-394, 2009.

8. Ha T, Hu Y, Liu L, Lu C, McMullen JR, Kelley J, Kao RL, Williams DL, Gao X and Li C: TLR2 ligands induce cardioprotection against ischaemia/reperfusion injury through a PI3K/Akt-dependent mechanism. Cardiovasc Res 87: 694-703, 2010.

9. Arslan F, Houtgraaf JH, Keogh B, Kazemi K, de Jong R, McCormack WJ, O'Neill LA, McGuirk P, Timmers L, Smeets MB, et al: Treatment with OPN-305, a humanized anti-toll-like receptor-2 antibody, reduces myocardial ischemia/reperfusion injury in pigs. Circ Cardiovasc Interv 5: 279-287, 2012.

10. Skyschally A, van Caster P, Iliodromitis EK, Schulz R, Kremastinos DT and Heusch G: Ischemic postconditioning: Experimental models and protocol algorithms. Basic Res Cardiol 104: 469-483, 2009. 
11. Staat P, Rioufol G, Piot C, Cottin Y, Cung TT, L'Huillier I, Aupetit JF, Bonnefoy E, Finet G, André-Fouët X and Ovize M: Postconditioning the human heart. Circulation 112: 2143-2148, 2005.

12. Zhang DW, Zhang L, Liu JG, Wang CL, Shi DZ and Chen KJ: Mechanisms of Chinese herbs combined with ischemic postconditioning in protecting myocardium of rats from ischemia-reperfusion injury. Zhong Xi Yi Jie He Xue Bao 8: 465-471, 2010 (In Chinese).

13. Wang $\mathrm{Y}, \mathrm{Ge} \mathrm{P}$, Yang $\mathrm{L}$, Wu $\mathrm{C}$, Zha $\mathrm{H}$, Luo $\mathrm{T}$ and Zhu $\mathrm{Y}$ : Protection of ischemic post conditioning against transient focal ischemia-induced brain damage is associated with inhibition of neuroinflammation via modulation of TLR 2 and TLR4 pathways. J Neuroinflammation 11: 15, 2014.

14. Zhong GQ, Tu RH, Zeng ZY, Li QJ, He Y, Li S, He Y and Xiao F: Novel functional role of heat shock protein 90 in protein kinase C-mediated ischemic postconditioning. J Surg Res 189: 198-206, 2014.

15. Sheedy FJ and O'Neill LA: Adding fuel to fire: MicroRNAs as a new class of mediators of inflammation. Ann Rheum Dis 67 (Suppl 3): iii50-iii55, 2008.

16. Wu CJ and Lu LF: MicroRNA in immune regulation. Curr Top Microbiol Immunol 410: 249-267, 2017.

17. Wojciechowska A, Braniewska A and Kozar-Kamińska K: MicroRNA in cardiovascular biology and disease. Adv Clin Exp Med 26: 865-874, 2017.

18. Hajibabaie F, Kouhpayeh S, Mirian M, Rahimmanesh I, Boshtam M, Sadeghian L, Gheibi A, Khanahmad H and Shariati L. MicroRNAs as the actors in the atherosclerosis scenario. J Physiol Biochem 76: 1-12, 2020.

19. Li Y, Lu J, Bao X, Wang X, Wu J, Li X and Hong W: MiR-499-5p protects cardiomyocytes against ischaemic injury via anti-apoptosis by targeting PDCD4. Oncotarget 7: 35607-35617, 2016.

20. Jia Z, Wang J, Shi Q, Liu S, Wang W, Tian Y, Lu Q, Chen P, Ma K and Zhou C: SOX6 and PDCD4 enhance cardiomyocyte apoptosis through LPS-induced miR-499 inhibition. Apoptosis 21: 174-183, 2016

21. Zhu J, Yao K, Wang Q, Guo J, Shi H, Ma L, Liu H, Gao W, Zou Y and Ge J: Ischemic postconditioning-regulated miR-499 protects the rat heart against ischemia/reperfusion injury by inhibiting apoptosis through PDCD4. Cell Physiol Biochem 39: 2364-2380, 2016.

22. Livak KJ and Schmittgen TD: Analysis of relative gene expression data using real-time quantitative PCR and the 2(-Delta Delta $\mathrm{C}(\mathrm{T})$ ) method. Methods 25: 402-408, 2001

23. Sheibani M, Faghir-Ghanesefat H, Dehpour S, Keshavarz-Bahaghighat H, Sepand MR, Ghahremani MH, Azizi Y, Rahimi N and Dehpour AR: Sumatriptan protects against myocardial ischaemia-reperfusion injury by inhibition of inflammation in rat model. Inflammopharmacology 27: 1071-1080, 2019

24. Sakata Y, Dong JW, Vallejo JG, Huang CH, Baker JS, Tracey KJ, Tacheuchi O, Akira S and Mann DL: Toll-like receptor 2 modulates left ventricular function following ischemia-reperfusion injury. Am J Physiol Heart Circ Physiol 292: H503-H509, 2007.

25. Selejan S, Poss J, Walter F, Hohl M, Kaiser R, Kazakov A, Böhm M and Link A: Ischaemia-induced up-regulation of Toll-like receptor 2 in circulating monocytes in cardiogenic shock. Eur Heart J 33: 1085-1094, 2012.

26. Arslan F, Smeets MB, O'Neill LA, Keogh B, McGuirk P, Timmers L, Tersteeg C, Hoefer IE, Doevendans PA, Pasterkamp G and de Kleijn DP: Myocardial ischemia/reperfusion injury is mediated by leukocytic toll-like receptor-2 and reduced by systemic administration of a novel anti-toll-like receptor-2 antibody. Circulation 121: 80-90, 2010.

27. Mersmann J, Berkels R, Zacharowski P, Tran N, Koch A Iekushi K, Dimmeler S, Granja TF, Boehm O, Claycomb WC and Zacharowski K: Preconditioning by toll-like receptor 2 agonist Pam3CSK4 reduces CXCL1-dependent leukocyte recruitment in murine myocardial ischemia/reperfusion injury. Crit Care Med 38: 903-909, 2010.
28. Chatterjee PK: Cardiac preconditioning by specific ligands of Toll-like receptors: Is it wither or whither? Crit Care Med 38: 1003-1004, 2010.

29. Dong JW, Vallejo JG, Tzeng HP, Thomas JA and Mann DL: Innate immunity mediates myocardial preconditioning through Toll-like receptor 2 and TIRAP-dependent signaling pathways. Am J Physiol Heart Circ Physiol 298: H1079-H1087, 2010.

30. Vilahur $\mathrm{G}$ and Badimon L: Ischemia/reperfusion activates myocardial innate immune response: The key role of the toll-like receptor. Front Physiol 5: 496, 2014.

31. Jin C, Cleveland JC, Ao L, Li J, Zeng Q, Fullerton DA and Meng X: Human myocardium releases heat shock protein 27 (HSP27) after global ischemia: The proinflammatory effect of extracellular HSP27 through toll-like receptor (TLR)-2 and TLR4. Mol Med 20: 280-289, 2014.

32. Jong WM, Ten Cate H, Linnenbank AC, de Boer OJ, Reitsma PH, de Winter RJ and Zuurbier CJ. Reduced acute myocardial ischemia-reperfusion injury in IL-6-deficient mice employing a closed-chest model. Inflamm Res 65: 489-499, 2016.

33. De Jesus NM, Wang L, Lai J, Rigor RR, Francis Stuart SD, Bers DM, Lindsey ML and Ripplinger CM: Antiarrhythmic effects of interleukin 1 inhibition after myocardial infarction. Heart Rhythm 14: 727-736, 2017

34. Kharbanda RK, Mortensen UM, White PA, Kristiansen SB, Schmidt MR, Hoschtitzky JA, Vogel M, Sorensen K, Redington AN and MacAllister R: Transient limb ischemia induces remote ischemic preconditioning in vivo. Circulation 106: 2881-2883, 2002

35. Przyklenk K, Bauer B, Ovize M, Kloner RA and Whittaker P: Regional ischemic 'preconditioning' protects remote virgin myocardium from subsequent sustained coronary occlusion. Circulation 87: 893-899, 1993.

36. Wang S, Zhang F, Zhao G, Cheng Y, Wu T, Wu B and Zhang YE: Mitochondrial PKC- $\varepsilon$ deficiency promotes I/R-mediated myocardial injury via GSK3 $\beta$-dependent mitochondrial permeability transition pore opening. J Cell Mol Med 21: 2009-2021, 2017.

37. Zheng H, Liu J, Liu C, Lu F, Zhao Y, Jin Z, Ren H, Leng X, Jia J, $\mathrm{Hu} \mathrm{G}$, et al: Calcium-sensing receptor activating phosphorylation of PKC $\delta$ translocation on mitochondria to induce cardiomyocyte apoptosis during ischemia/reperfusion. Mol Cell Biochem 358: 335-343, 2011.

38. Cario E, Gerken G and Podolsky DK: Toll-like receptor 2 enhances ZO-1-associated intestinal epithelial barrier integrity via protein kinase C. Gastroenterology 127: 224-238, 2004.

39. Park DW, Lee HK, Lyu JH, Chin H, Kang SW, Kim YJ, Bae YS and Baek SH: TLR2 stimulates ABCA1 expression via PKC- $\eta$ and PLD2 pathway. Biochem Biophys Res Commun 430: 933-937, 2013.

40. Jiang SY, Wei CC, Shang TT, Lian Q, Wu CX and Deng JY: High glucose induces inflammatory cytokine through protein kinase C-induced toll-like receptor 2 pathway in gingival fibroblasts. Biochem Biophys Res Commun 427: 666-670, 2012.

41. Xin Y, Yang C and Han Z: Circulating miR-499 as a potential biomarker for acute myocardial infarction. Ann Transl Med 4: 135,2016

42. Liu X, Fan Z, Zhao T, Cao W, Zhang L, Li H, Xie Q, Tian Y and Wang B: Plasma miR-1, miR-208, miR-499 as potential predictive biomarkers for acute myocardial infarction: An independent study of Han population. Exp Gerontol 72: 230-238, 2015.

43. Qin H, Chen GX, Liang MY, Rong J, Yao JP, Liu H and Wu ZK: The altered expression profile of microRNAs in cardiopulmonary bypass canine models and the effects of mir-499 on myocardial ischemic reperfusion injury. J Transl Med 11: 154, 2013.

This work is licensed under a Creative Commons Attribution-NonCommercial-NoDerivatives 4.0 International (CC BY-NC-ND 4.0) License. 